Meta

Journal des traducteurs

Translators' Journal

\title{
Le PPBS et sa terminologie
}

\section{Jean Delisle}

Volume 15, numéro 4, décembre 1970

URI : https://id.erudit.org/iderudit/002519ar

DOI : https://doi.org/10.7202/002519ar

Aller au sommaire du numéro

Éditeur(s)

Les Presses de l'Université de Montréal

ISSN

0026-0452 (imprimé)

1492-1421 (numérique)

Découvrir la revue

Citer cet article

Delisle, J. (1970). Le PPBS et sa terminologie. Meta, 15(4), 222-224.

https://doi.org/10.7202/002519ar d'utilisation que vous pouvez consulter en ligne.

https://apropos.erudit.org/fr/usagers/politique-dutilisation/ 


\section{LE PPBS ET SA TERMINOLOGIE}

Un nouveau système d'élaboration du budget national a été lancé aux États-Unis en 1963. La France a adopté le système en 1969 et le Canada en 1970. Il s'agit du PPBS (Planning-Programming-Budgeting System) que l'on a rendu à Ottawa par «Planification-programmation-budgétisation» et en France par «Prospective-plan-budget». Dans ce dernier pays, cependant, le système a été baptisé la « Rationalisation des choix budgétaires »; légèrement modifiée, la RCB s'inspire en droite ligne du PPBS.

Le PPBS est un système de budgétisation (budgeting) axé sur la planification (planning). \& Il part d'un effort de prospective concernant des objectifs et, au moyen de l'analyse, traduit les programmes en propositions budgétaires ${ }^{1}$. $\gg \mathbf{L a}$ mise en application du PPBS ou de la RCB comporte trois phases principales.

La première est l'explicitation des objectifs (planning). Cette étape s'appelle aussi en français planification, prospective ou stratégie. Il ne faut pas confondre planning et programming que nous rencontrerons plus loin. «The planning is the production of the range of meaningful potentials for selection of courses of action through a systematic consideration of alternatives ${ }^{2}$. C 'est certainement l'opération la plus délicate, car elle consiste à faire l'appréhension des objectifs (objectives) et la reconnaissance des cheminements logiques qui les concrétisent. Désormais, les budgets ne seront plus élaborés pour un an, mais ils seront pluriannuels, le budget annuel n'étant qu'une tranche d'un budget septennal, quinquennal, quadriennal ou encore triennal. C'est ce qui caractérise le PPBS : les objectifs sont des éléments intégrés au budget et non plus de simples boussoles. On conçoit alors facilement qu'il faille actualiser (to discount) le développement économique et social de la nation et sélectionner des projets en fonction des ressources disponibles. "L'actualisation (discount, discounting) est un système de pondération dont l'objet est de ramener les valeurs futures à leur valeur

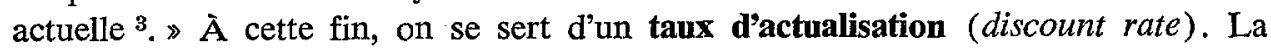
première phase est donc d'ordre stratégique. Par le processus décisionnel (decisionmaking process), les dirigeants choisissent des programmes convergeant vers ces objectifs.

La deuxième phase est la programmation ou le plan (programming). Elle consiste à faire l'analyse et la sélection des programmes. "The programming is a specific determination of manpower, material, and facilities necessary for accomplishing a program ${ }^{4}$.» De la stratégie générale (planning), on passe donc à la tactique (programming), c'est-à-dire l'analyse des programmes (program analysis)

1. Charles Schultz, cité par Marc Clairvois, dans \& La révolution du PPBS », l'Expansion; 1968, p. 108.

2. David Novick, Program Budgeting, Cambridge (Mass.), Harvard University Press, 1965, p. 91.

3. Ce néologisme a été relevé dans la revue Finances et développement, no 3 (1969), citée dans l'Actualité terminologique, vol. $2, \mathrm{n}^{\circ} 10$ (déc. 1969), p. 4.

4. David Novick, Program Budgeting, p. 91. 
dominée par les techniques mathématiques ${ }^{5}$. L'analyse permet d'aborder les problèmes de façon systématique et rationnelle. On procède surtout à des analyses avantages-coûts ou cô̂ts-bénéfices (benefit-cost analysis) et à des analyses coûtsefficacité (cost-utility ou cost-effectiveness analysis). Une des notions d'économique les plus importantes est sans doute celle d'options ou de choix (trade-off, alternatives). «Un avantage particulièrement pratique de la notion d'options est de permettre de bien déterminer le rôle des différents genres de politiques économiques ${ }^{6}$. $\gg$ A cette fin, on établit des courbes d'options (trade-off curves) et détermine des zones d'options (trade-off zones). Actuellement, le sens de trade-off couvre par extension celui de choix à faire entre un objectif économique et un objectif politique, comme en fait preuve ce passage du Time : «But now that the U.S. industrial and social system is delivering such «disproducts» as pollution and racial tension [...] many Americans feel they have been swindled in the trade-off ${ }^{7}$. » Quant au coût d'option (alternative cost), il exprime ce qu'il a fallu sacrifier par suite du choix d'un programme. La recherche opérationnelle (operations research) consiste à analyser un grand nombre de problèmes complexes où les interactions sont nombreuses et elle fournit le maximum d'informations quantitatives sur les incidences des différentes options possibles. Par cette deuxième phase, on est passé de l'abstrait au concret, de la réflexion à l'action. Car le programme est « une activité ou une série d'activités considérée comme une entité opérationnelle, budgétaire ou administrative ${ }^{8} \gg$ qui ne vise qu'à réaliser un objectif. Le programme peut se subdiviser en sous-programmes (subprograms) et même en sous-sous-programmes ( $s u b$-subprograms), jusqu'à des unités élémentaires de structure (building blocks); ces unités élémentaires peuvent prendre le nom de «projets» et sont des tâches bien déterminées dans l'espace et le temps. Elles assurent en outre au système une certaine flexibilité. Les moyens ou ressources (resources) sont les intrants (input) d'un programme, les facteurs nécessaires pour l'exécution d'un programme. Quant au but ou extrant (goal, output), il est la mesure physique d'un objectif et il le traduit de façon opératoire. C'est le produit final résultant de l'application d'un programme.

La troisième et dernière phase est évidemment le plan de dépenses ou budgétisation (budgeting) ou encore la budgétisation des programmes (program budgeting). "Elle traduit en termes monétaires l'ensemble des programmes retenus, et elle mesure le degré de réalisation des objectifs qu'ils desservent dans l'unité physique ou monétaire appropriée à leur nature. Elle conduit à la confection de plans physiques et financiers se déroulant sur plusieurs années ${ }^{9}$.

5. L'emploi de termes militaires comme stratégie et tactique s'explique par le fait que le PPBS a été introduit aux Etats-Unis, au secrétariat d'Etat à la Défense, par M. McNamara, en 1961. Mais il y a véritablement un rapport entre le planning (stratégie) et le programming (tactique); selon Bénac, la stratégie, à l'échelle la plus vaste, prépare et dirige l'ensemble des opérations de guerre, tandis que la tactique, dans une situation donnée, face à l'ennemi, est l'art de disposer et de manouvrer les troupes sur le terrain.

6. Conseil économique du Canada (troisième exposé annuel), les Prix, la productivité et l'emploi, Ottawa, Imprimeur de la Reine, 1966, p. 159.

7. Time, 23 avril 1970 , p. 67.

8. Jean-Claude Bluet, "Le Planning-Programming-Budgeting System ", Metra, VII, 3 : 409-410.

9. Ibid., p. 401. 
Le PPBS ne tardera sûrement pas à envahir tout le domaine administratif. Il est intéressant de noter que sa mise en application exige une terminologie uniformisée pour toutes les divisions administratives. Le PPBS entraînera donc une «planification de la terminologie » dans les bureaux de traduction.

\section{MINI-GLOSSAIRE DU PPBS}

alternative

alternative cost

benefit-cost analysis

budgeting

building block

cost-effectiveness analysis

cost-utility analysis

decision-making process

discount

discount rate

discount (to)

discounting

goal

input

objectives

operations research

output

planning

PPBS

program analysis

program budgeting

programming

RCB

resources

subprogram

sub-subprogram

trade-off

trade-off curve

trade-off zone

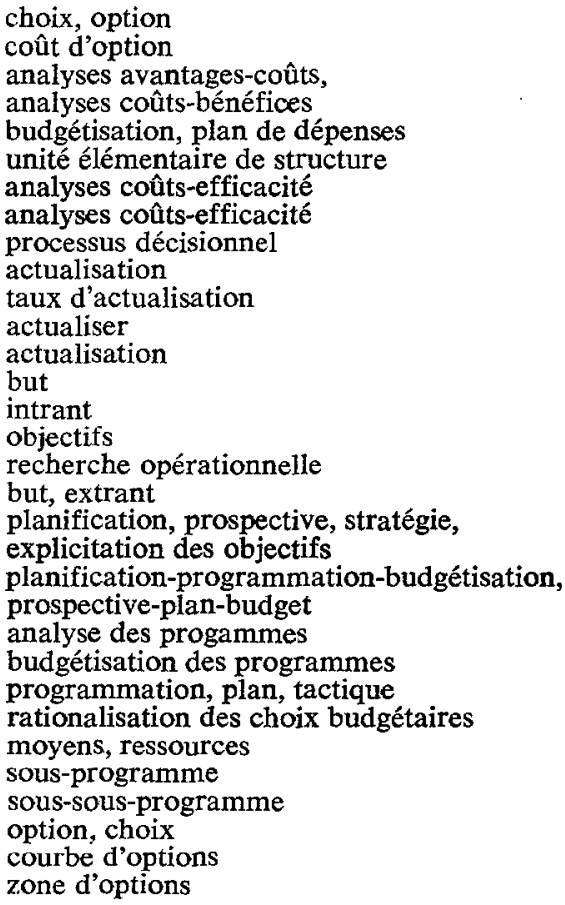

JEAN DELISLE 